BULL. AUSTRAL. MATH. SOC.

VOL. $20(1979), 253-257$.

\title{
Weak star separability
}

\section{EN. Dancer and Brailey Sims}

\begin{abstract}
For a Banach space $X$, Susumu Okada raised the question of whether the unit ball of the dual space $X^{*}$ is weak* separable if $X^{*}$ is weak* separable. The problem occurred in the theory of manifolds modelled on locally convex spaces. We answer the question in the negative but show that it is true for particular types of spaces.
\end{abstract}

Our basic purpose is to present a counter-example to the contention:

If $X^{*}$ is weak* separable, then $B\left[X^{*}\right]$ is weak* separable.

$X$ is an infinite dimensional Banach space, $X^{*}$ its dual, and $B\left[X^{*}\right]$ the unit ball in $X^{*}$; that is, $\left\{f \in X^{*}:\|f\| \leq 1\right\}$. We begin by noting that the converse is trivially true.

(1) If $B\left[X^{*}\right]$ is weak* separable then so too is $X^{*}$.

Proof. Let $\left(f_{n}\right)$ be a $w^{*}$ dense sequence in $B\left[X^{*}\right]$ and let $N$ be any $w^{*}$ open set in $X^{*}$. Choose $f \in N$ and rational $q>\|f\|$. Then $q^{-1} N$ is a $w^{*}$ open set intersecting $B\left[X^{*}\right]$. Thus there exists $f_{n} \in q^{-I} N$ and so we conclude that the countable set of rational multiples of elements in $\left(f_{n}\right)$ is $w^{*}$ dense in $X^{*}$.

Examples of spaces in which $B\left[X^{*}\right]$ is weak* separable include:

(i) Duals of separable spaces. The relative $w^{*}$ topology on the $w^{*}$ compact set $B\left[X^{*}\right]$ is a metric topology (Dunford and Schwartz [2], p. 426).

Received 13 March 1979. Communicated by Sadayuki Yamamuro. 
(ii) Biduals of separable spaces. For example $Z_{\infty}$ or $l_{\infty}^{*}$. Let $\left(x_{n}\right)$ be a norm dense sequence in $B[X]$; we show $\left(x_{n}\right)$ is $w^{*}$ dense in $B\left[X^{* *}\right]$. For any relative $\omega^{*}$ open subset $N$ of $B\left[X^{*}\right]$, there exists, by Goldstine's Theorem (Dunford and Schwartz [2], p. 424), an $x \in B[X]$ with $\hat{x} \in N$. By the relative strengths of the topologies, for some $r>0, B_{r}(\hat{x}) \subset N$. The argument is completed by observing that $B_{r}(x)$ contains an element of $\left(x_{n}\right)$, and hence $N$ contains an element of $\left(\hat{x}_{n}\right)$.

(iii) Reflexive spaces if and only if they are separable. The 'if' part is obvious. On the other hand, if $B\left[X^{*}\right]$ is $\omega^{*}$ separable where $X$ is reflexive, then by (1), $X^{*}$ is $w^{*}$ separable. Since the $w^{*}$ and $w$ topologies coincide we have, by Mazur (Rudin [6], p. 64), that $X^{*}$ is separable.

Note. This argument also shows that the contention is true in reflexive spaces.

We next obtain conditions equivalent to the two properties in the contention. This allows the contention to be reformulated in a variety of ways.

We first consider $B\left[X^{*}\right]$ weak ${ }^{*}$ separable.

(2) The following are equivalent:

(a) $B\left[X^{*}\right]$ is weak* separable;

(b) $B\left[X^{*}\right]$ contains a countable strictly norming subset of $X$;

(c) $X^{*}$ has a separable subspace which strictly norms $X$;

(d) $X$ is isometric to a subspace of $\eta_{\infty}$.

Proof. $(a) \Rightarrow(b)$. Let $\left(f_{n}\right)$ be a $w^{*}$ dense sequence in $B\left[X^{*}\right]$. Then, for each $x \in X$ with $\|x\|=1$ and $\varepsilon>0$, there exists an element of $\left(f_{n}\right)$ in the non-empty relatively $\omega^{*}$ open subset $\left\{f \in B\left[X^{\star}\right]: f(x)>1-\varepsilon\right\}$.

$(b) \Rightarrow(a)$. Let $\left(g_{n}\right)$ be a sequence in $B\left[X^{*}\right]$ which strictly norms $X$; then $\overrightarrow{\operatorname{co}^{*}}\left(g_{n}\right)$ has the rational convex combinations of $\left(g_{n}\right)$ as a countable $w^{*}$ dense subset. Thus it suffices to show $\overrightarrow{c o}^{*}\left(g_{n}\right)=B\left[X^{*}\right]$. 
If there exists $f_{0} \in B\left[X^{*}\right] \backslash \overrightarrow{c o}^{\omega^{*}}\left(g_{n}\right)$, then, by the separation theorem (Day [1], p. 24), there is a $w^{*}$ continuous functional $\hat{x}$ with $\|x\|=1$ such that $f_{0}(x)>\sup \left\{f(x): f \in \overrightarrow{c o}^{\omega^{*}}\left(g_{n}\right)\right\}$, contradicting the fact that $\left(g_{n}\right)$ strictly norms $X$.

$(b) \Rightarrow(c)$ and $(c) \Rightarrow(b)$ are obvious.

$(b) \Rightarrow(d)$. Let $\left(g_{n}\right)$ be as in the proof of $(b) \Rightarrow(a)$, and consider the mapping $\phi: X \rightarrow z_{\infty}: x \mapsto\left(g_{1}(x), g_{2}(x), \ldots, g_{n}(x), \ldots\right)$. Clearly $\phi$ is linear. Further, $\|\phi(x)\|_{\infty}=\sup _{n} g_{n}(x)=\|x\|$.

$(d) \Rightarrow(b)$. Let $\phi$ be an isometry from $X$ into $z_{\infty}$. Define $g_{n}$ by $g_{n}(x)=\phi(x)(n)$, the $n$th component of $\phi(x)$. Clearly $g_{n} \in B\left[X^{*}\right]$ and $\left\{g_{n}\right\}$ is strictly norming for $X$.

COROLLARY 3. If $X^{*}$ is non separable and $B\left[X^{*}\right]$ is the norm closed convex hull of points at which the relative weak* and norm topologies on $B\left[X^{*}\right]$ agree, then $B\left[X^{*}\right]$ is not weak* separable.

Proof. Assume $B\left[X^{*}\right]$ is weak* separable, then, by (2) (c), $X^{*}$ contains a separable subspace $M$ which strictly norms $X$. Since $M$ is proper there is a point $f$ of $B\left[X^{*}\right]$ at which the relative $\omega^{*}$ and norm topologies agree but which is not in $B[M]$. Now $B[M]$ is $\omega^{*}$ dense in $B\left[X^{*}\right]$, so there exists a net $\left(f_{\alpha}\right)$ in $B[M]$ which converges $\omega^{*}$ to $f$; but. then $\left\|f_{\alpha}-f\right\| \rightarrow 0$, so $f \in B[M]$, a contradiction.

Excomples of spaces which satisfy the condition of Corollary 3 are:

(i) $X^{*}$, where $X$ satisfies condition (**) (Namioka [5]); and hence in particular

(ii) Locally uniformly rotund dual spaces;

(iii) duals of spaces with Fréchet differentiable norms.

If $X$ has a Fréchet differentiable norm, then by the Bishop-Phelps theorem and Šmulian's dual characterization of points of strong differentiability, the $\omega^{*}$ strongly exposed points of $B\left[X^{*}\right]$ are dense in the boundary. Further, at such points the relative $w^{*}$ and norm topologies agree. 
(iv) dual spaces for which $B\left[X^{*}\right]$ is the norm closed convex hull of the $w^{*}$ denting points (Sims); in particular duals of spaces with the Mazur intersection property (Giles, Gregory, and Sims [3]).

Thus any non-separable, $w^{*}$ separable, dual space satisfying any of the preceding conditions would provide a counter-example to the contention.

Although we shall not need it, a result, similar to (2) for $X^{*} w^{*}$ separable is possible.

(4). The folzowing are equivalent:

(a) $X^{*}$ is $w^{*}$ separable;

(b) $X^{*}$ (and hence $B\left[X^{*}\right]$ ) contains a countable total subset;

(c) $X^{*}$ has a separable total subspace;

(d) there is a one-to-one linear continuous mapping from $X$ into $z_{\infty}$.

Proof. The proof is similar to that for (2). We illustrate it with

$(b) \Rightarrow(a)$. Let $\left(f_{n}\right)$ be a total sequence in $X^{*}$. Then the rational linear combinations of $\left(f_{n}\right)$ are dense in $M=\overrightarrow{\operatorname{span}}^{*}\left(f_{n}\right)$. Further, $M=X^{*}$. Otherwise, by the Hahn-Banach Theorem there is a $w^{*}$ continuous functional $\hat{x} \neq 0$ such that $M \subseteq$ ker $\hat{x}$; thus $f_{n}(x)=0$ for all $n$. Contradicting the fact, $\left(f_{n}\right)$ is total.

COUNTER EXAMPLE 5. Johnson and Lindenstrauss [4], Example 1, p. 222, have constructed a space $U$ having the following relevant properties.

(a) $U^{*}$ is isomorphic to $l_{1} \oplus l_{2}(\Gamma)$ where $\Gamma$ is a set of cardinality that of the continuum. So $U^{*}$ is non-separable.

(b) $U^{*}$ is weak* separable;

(c) $U$ is not isomorphic to a subspace of $Z_{\infty}$. It is also claimed that

(d) $U$ has an equivalent Fréchet differentiable norm (indeed, that $U^{*}$ admits an equivalent locally uniformly rotund dual norm). 
Thus by (2) (d) we have

$U^{*}$ is weak* separable, but for no equivalent dual norm on $U^{*}$ is $B\left[U^{*}\right]$ weak* separable.

REMARK. Recently, some doubt has been cast on the validity of the claim (d) above (Yost [8]). However this does not apply to the properties of $U$ essential to our purpose.

\section{References}

[1] Mahlon M. Day, Normed Zinear spaces, 3rd ed. (Ergebnisse der Mathematik und ihrer Grenzgebiete, 21. Springer-Verlag, Berlin, Heidelberg, New York, 1973).

[2] Nelson Dunford and Jacob T. Schwartz, Linear operators. Part I: General theory (Pure and Applied Mathematics, 7. Interscience, New York, London, 1958).

[3] J.R. Giles, D.A. Gregory, and Brailey Sims, "Characterisation of normed linear spaces with Mazur's intersection property", Buzl. Austral. Math. Soc. 18 (1978), 105-123.

[4] W.B. Johnson and J. Lindenstrauss, "Some remarks on weakly compactly generated Banach spaces", Israel J. Math. 17 (1974), 219-230.

[5] I. Namioka, "Separate continuity and joint continuity", Pacific J. Math. 51 (1974), 515-531.

[6] Walter Rudin, Functional analysis (McGraw-Hill, New York, St. Louis, San Francisco, Düsseldorf, Johannesburg, Kuala Lumpur, London, Mexico, Montreal, New Delhi, Panama, Rio de Janeiro, Singapore, Sydney, Toronto, 1973).

[7] Brailey Sims, "Local agreement of the relative weak* and strong topologies on weak* compact sets and weak*-denting points", submitted.

[8] David Thomas Yost, "Spaces which admit convex norms" (MSc thesis, Australian National University, Canberra, 1977).

Department of Mathematics, University of New England, Armidale, New South Wales. 